\title{
A VISCOELASTIC-AWARE EXPERIMENTALLY-DERIVED MODEL FOR ANALOG RF MEMS VARACTORS
}

\author{
Hao-Han Hsu and Dimitrios Peroulis \\ Birck Nanotechnology Center, Purdue University, West Lafayette, IN 47907, USA
}

\begin{abstract}
In this paper we present, for the first time, an experimentally-extracted model for the spring constant and tuning range of an analog RF-MEMS varactor that includes viscoelastic effects in RF-MEMS devices. By utilizing a bi-state bias condition with one state lasting 60 minutes and the other 1 minute, this model focuses on capturing the true electromechanical behavior of the varactor. An experimental setup with very high long-term accuracy is created to measure capacitance of the varactor up to 1,370 hours. The impact of these effects and the effectiveness of the model are demonstrated on a tunableresonator loaded with $\mathrm{RF}-\mathrm{MEMS}$ varactors.
\end{abstract}

\section{INTRODUCTION}

Viscoelasticity is one of the most important areas of concern in the long-term operation of MEMS devices. It can be observed as the time dependency of deflection, spring constant, elastic modulus, and yield strength, among others, in MEMS devices $[1,2]$. Most thin-film metals that are widely employed in metallic RF-MEMS devices, including $\mathrm{Au}, \mathrm{Al}$, and $\mathrm{Ni}$, exhibit viscoelastic behavior. This behavior needs to be studied and modeled in order to achieve the required reliability and long-term controllability.

Vicker-Kirby et al. reported anelastic phenomena in MEMS cantilever accelerometers [3]. Si, Ni, and $\mathrm{Au}$ cantilevers were controlled electrostatically using a feedback circuit that sensed the tunneling current at the cantilever tip and applied an appropriate voltage to maintain a constant gap of $10 \AA$. This voltage decreased gradually within the measurement duration of 30 hours. This experiment also demonstrated that $\mathrm{Au}$ is most prone to creep among the three materials. Gilz et al. performed an experiment on an Al RF-MEMS switch [4]. It was pulled-in using electrostatic force and the deformation of the suspension beam was measured optically for 15 hours. A finite-element-analysis (FEA) simulation was conducted based on a measured macro-scale creep-response to verify the micro-scale measurement. However, discrepancies were found between the measurement and the FEA simulation. Yan et al. performed a stress-relaxation test on an Au thin-film using a bulge test technique [1]. The Au film was inflated pneumatically and the capacitance between the deformed Au-film and the

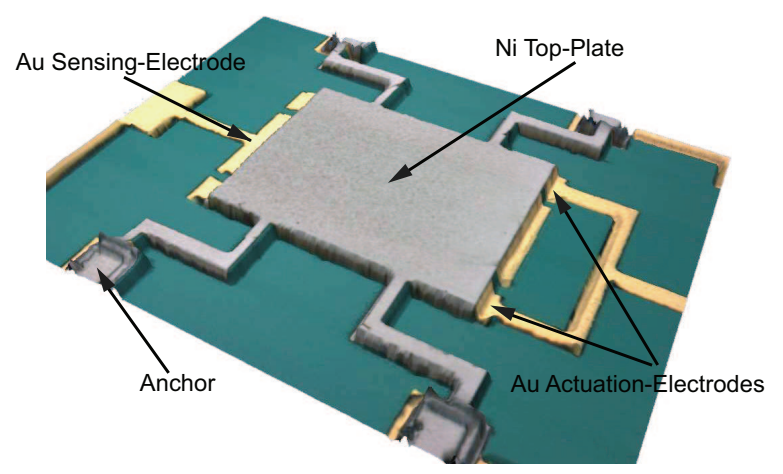

Figure 1. The confocal-microscopy image of the fabricated analog RF-MEMS varactor. The gap between the Ni plate and Au electrodes is $3 \mu \mathrm{m}$. There are no dielectric layers in this varactor.

electrode beneath was measured for 80 hours. A cyclic-loading test was also carried out to demonstrate the linearity of viscoelasticity in such film. The decrease of restoring force in the RF-MEMS switch was discussed based on the calculation and measurement of the bulged Au-film. Chasiotis et al. conducted an investigation into the strain-rate effects on the mechanical behvaior of nanocrystalline $\mathrm{Au}$ films [2]. Atomic force microscopy (AFM) and digital image correlation (DIC) were utilized in the tensile tests of dog-bone shaped specimens at various strain-rates. The decrease of the maximum strength, effective Young's modulus, and yield strength in the Au thin-films at low strain-rates was attributed to dislocation creep.

In this paper, we present an experimentallyextracted model that can accurately describe spring constant and tuning range of analog RF-MEMS varactors, based on capacitance measurements up to 1,370 hours. This measurement setup has been carefully established to achieve high accuracy and high long-term stability. A new parameter called dynamic spring constant is proposed to reveal the true electromechanical behavior of the analog RFMEMS varactor under the effect of viscoelasticity. The impact of viscoelasticity is demonstrated by applying the experimentally-extracted model to a tunable-resonator loaded with RF-MEMS varactors. This model and dynamic spring constant may improve the controllability of MEMS devices in long-term operation and lead to more reliable designs. 


\section{ANALOG RF-MEMS VARACTOR}

The confocal microscopy image of the analog RFMEMS varactor is shown in Fig. 1. The moving parts of the varactor consists of a $300-\mu \mathrm{m} \times 220-\mu \mathrm{m}$ $\mathrm{Ni}$ top-plate and 4 symmetric suspension-beams. Three Au electrodes can be found underneath the Ni plate. The bias voltage is applied to the two electrodes at the side, and the capacitance is sensed using the center electrode. The thickness and total length of one suspension beam is about $3 \mu \mathrm{m}$ and $200 \mu \mathrm{m}$ respectively. The actuation voltage is about $50 \mathrm{~V}$. Unlike switched RF-MEMS devices, there are no dielectric layers in this design. This eliminates the effect of dielectric charging and provides substantial insight into the viscoelastic behavior of ana$\log$ RF-MEMS devices.

The fabrication process of this $\mathrm{Ni}$ analog RFMEMS varactor is shown in Fig. 2. The varactor is built on a $p$-type high-resistivity silicon substrate with a 500-nm thick thermally-grown silicondioxide film. An 1- $\mu \mathrm{m}$ thick $\mathrm{Au}$-film is sputtered and lifted-off to define the bottom electrodes and electrical connections of the varactor in Fig. 2(a). The anchors are then patterned through a $3-\mu \mathrm{m}$ thick photoresist sacrificial layer in Fig. 2(b). The sacrificial layer is hard-baked at $190^{\circ} \mathrm{C}$ for 5 minutes. A seed-layer of 50-nm sputtered Ti and 30$\mathrm{nm}$ evaporated $\mathrm{Ni}$ is deposited on the whole sample in Fig. 2(c). A 6- $\mu \mathrm{m}$ thick photoresist layer is shaped to form the electroplating mold on the seed layer. The Ni electroplating is carried out in a nickel-sulfamate bath at a temperature of $50^{\circ} \mathrm{C}$ and a $\mathrm{pH}$ value of 4 . The average grain-size is about $50 \mathrm{~nm}$. A $3-\mu \mathrm{m}$ thick Ni layer is electroplated on the seed layer selectively based on the photoresist mold as shown in Fig. 2(d). The Ni and Ti seed layers are stripped with $\mathrm{HCl}$ : water $=1: 1$ and $\mathrm{HF}$ : water $=1: 20$ at room temperature respectively after the removal of the photoresist mold. The photoresist sacrificial layer is removed by immersion in photoresist-stripper-2000 at $75^{\circ} \mathrm{C}$ for 24 hours. Finally, the fabrication process is completed by drying in a critical-point-dryer (CPD) in Fig. 2(e).

\section{EXPERIMENTAL SETUP}

A commercial capacitance sensor, AD7746, from Analog Devices, Inc., is employed to perform lownoise, low-capacitance measurement on the ana$\log$ RF-MEMS varactors, which is wire-bonded and mounted on an Au-electroplated pin-grid-array (PGA) package. This AD7746 is a high-precision capacitance-to-digital converter (CDC) consisting of a 24-bit $\Sigma$ - $\Delta$ modulator and a third-order digital filter. This circuit feeds a $32 \mathrm{k}-\mathrm{Hz}$ square wave to the capacitor-under-test and converts the charge going through the capacitor to a binary code.

Two difficulties can be encountered during the observation of viscoelastic behavior. First, sub-

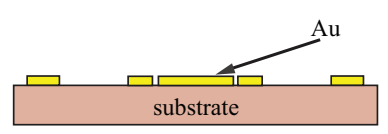

(a)

(b)

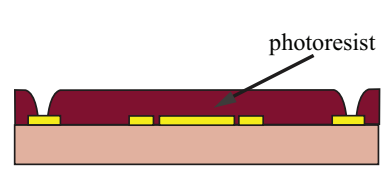

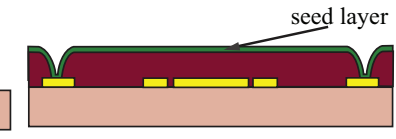

(c)

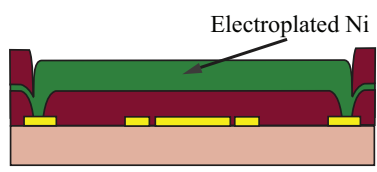

(d)

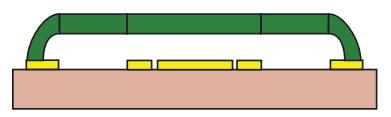

(e)

Figure 2. Fabrication process of the Ni analog RFMEMS varactor.

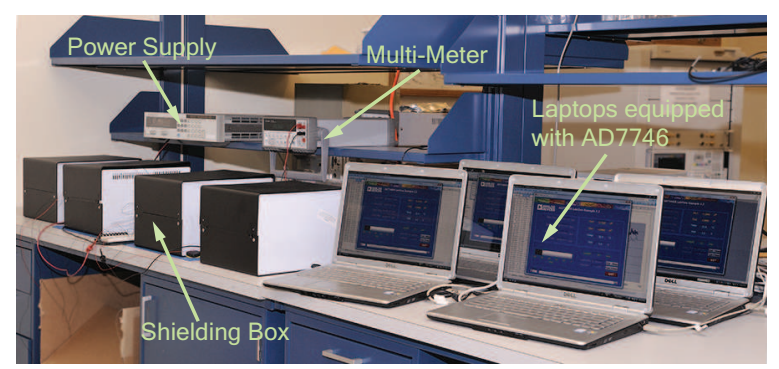

Figure 3 . The experimental setup consists of opaque shielding boxes, laptops equipped with AD7746, a high-voltage power supply, and a multi-meter.

femto-farad accuracy is required. Second, the measurement drifting needs to be minimized to reveal the true viscoelastic movement. The noise is the limiting factor of the measurement uncertainty. In this work, each data point is the average of 100 acquired-samples and the uncertainty of such a point is defined as one standard-deviation of these 100 samples. The noise can be lowered by utilizing an opaque electrically-grounded shielding box and coaxial cables. The measurement uncertainty is less than $200 \mathrm{aF}$ in Fig. 4(a).

The long-term drift of the experimental setup needs to be characterized to validate that the measured capacitance-change is due to the nature of viscoelasticity and not the artificial drift of the experimental setup. A long-term drift test is carried out by measuring the capacitance of the ana$\log$ RF-MEMS varactor without applying any bias for 1,500 hours in Fig. 4(b). It's confirmed that the effect of the long-term drift can be neglected in this work. The overall drift is less than $4 \mathrm{fF}$ over 1,500 hours, which is about $1 \%$ of the varactor capacitance at 1,000 hour and beyond.

\section{RESULTS AND DISCUSSION}

The analog RF-MEMS varactors are measured using a bi-state bias condition. In the first state a constant bias-voltage $V_{\text {bias }}$ is applied to the varactor for 60 minutes. In the second state the bias 


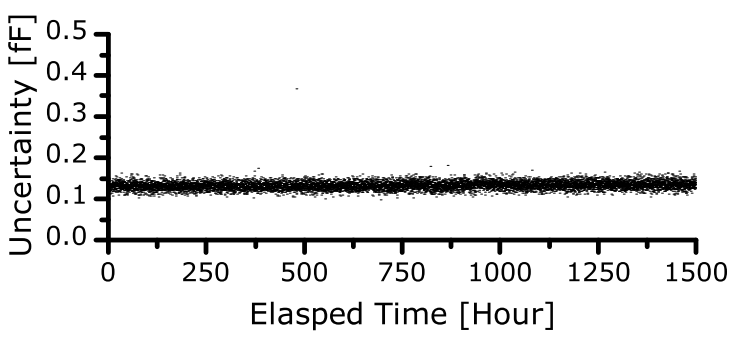

(a)

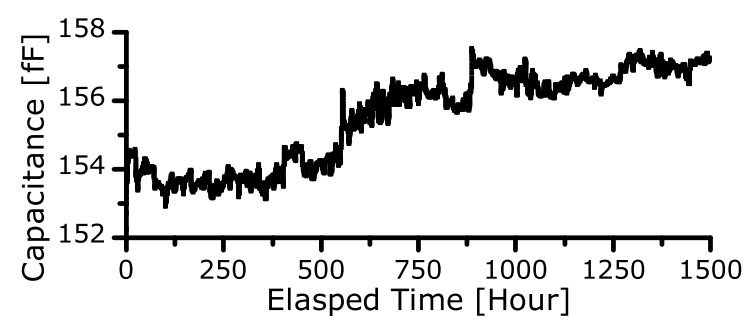

(b)

Figure 4. (a) The measurement uncertainty. The measured long-term drift is less than $4 \mathrm{fF}$ over 1,500 hours.

voltage is removed for 1 minute. In Fig. 5(a) the biased curve represents the capacitance measured in the first bias state when $V_{\text {bias }}$ is $40 \mathrm{~V}$. The capacitance measured in the second state when $V_{\text {bias }}$ is removed is shown in the unbiased curve. A 3-D electrostatic model of the varactor that contains all the electrodes, pads, and the substrate is established in a FEA tool [5]. The gap in Fig. 5(b) and the electrostatic force (not shown) are extracted using this model. The parasitics and the fringing field are included in this FEA model, and thus a more accurate extraction of the gap and electrostatic force than the parallel-plate model is obtained. The measured data and also the extracted data can be modeled using a series of decaying exponents by curve-fitting,

$$
y(t)=A_{o}+\sum_{i=1}^{n} A_{i} e^{-t / \tau_{i}}
$$

where $A_{i}$ and $\tau_{i}$ are constant.

In contrast to the traditional point of view that the capacitance of varactor returns to the initial capacitance $C_{o}$ after the bias is removed, the capacitance $C_{\text {unbias }}$ is determined by the loading history and stays between $C_{b i a s}$ and $C_{o}$. This phenomenon is largely due to the viscoelasticity of the material and not to dielectric charging because there are no dielectric layers between the varactor.

\section{Dynamic Spring Constant}

The conventional spring constant in Fig. 6(a) is given by

$$
K_{\text {conv }}(t)=\frac{F(t)}{\left(g_{o}-g(t)\right)}
$$

where $F(t)$ is the FEA-extracted force, $g_{o}$ is the inital gap, and $g(t)$ is the gap at time $t$. An assump-

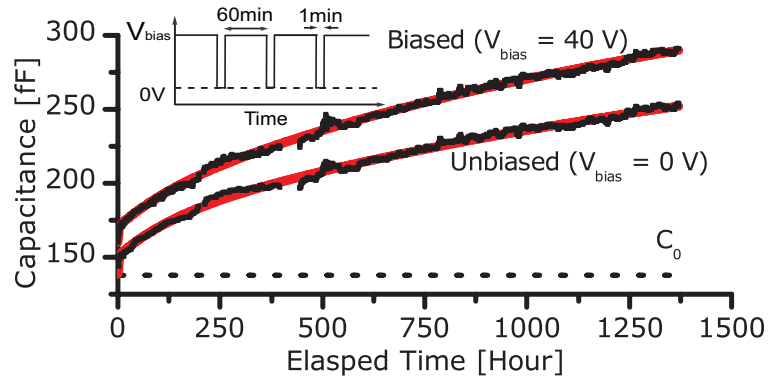

(a)

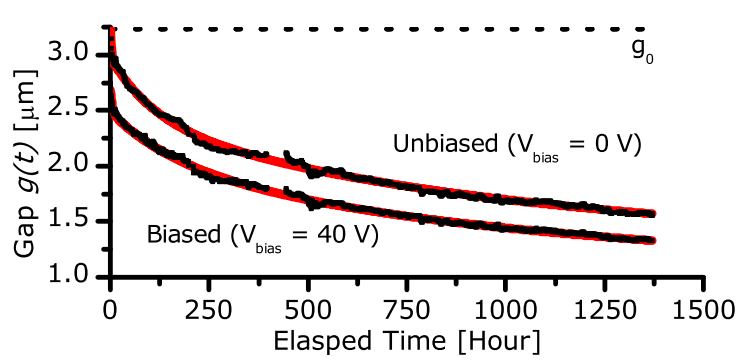

(b)

Figure 5. The measured (black) and fitted (red) results of the analog RF-MEMS varactor. (a) The measured capacitance. $C_{o}$ is the initial capacitance. (b) The FEA-extracted gap. $g_{o}$ represents the initial gap.

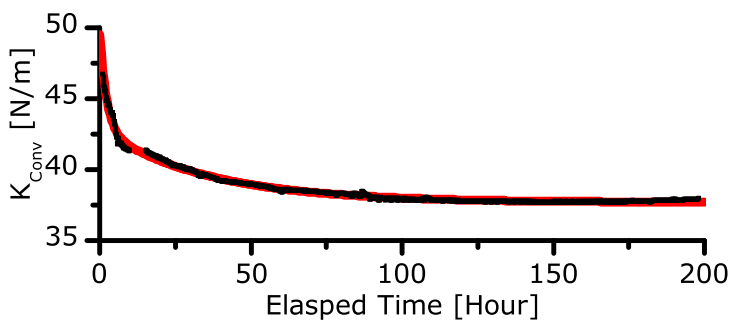

(a)

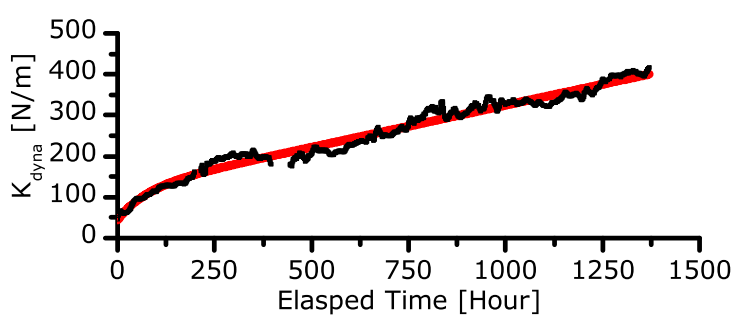

(b)

Figure 6. The measured (black) and fitted (red) spring constant. (a) Conventional spring constant. (b) Dynamic spring constant.

tion is made that the varactor reverts to the initial gap $g_{o}$ when the bias voltage is removed. This assumption is not true according to the extracted gap in Fig. 5(b). The conventional spring constant is not sufficient to describe the viscoelastic behavior of the analog RF-MEMS varactor. Nevertheless, the extracted conventional spring constant decays over time and is consistent with the measurement results of relaxation modulus in other literature $[1,6]$. 
In order to correctly capture the actual behavior of the analog RF-MEMS varactor, a new parameter called dynamic spring constant is defined,

$$
K_{\text {dyna }}(t)=\frac{F(t)}{\left(g_{\text {unbias }}(t)-g(t)\right)}
$$

where $F(t)$ is the force, $g(t)$ is the gap at time $t$, and $g_{\text {unbias }}(t)$ is the gap at time $t$ if the bias voltage is removed. The force required for the desired deflection in the varactor can be correctly derived using this spring constant since the viscoelastic behavior that the varactor doesn't revert to the initial gap $g_{o}$ is considered. This spring constant along with the function $g_{\text {unbias }}(t)$ provides the ability to accurately control the gap of the varactor in the longterm operation. The measurement shows that the dynamic spring constant may rise by as much as 8 times after the operation of 1,370 hours. The cause of the increase in this spring constant is related to strain-hardening.

\section{Tuning Range}

The impact of viscoelastic behavior on tuning range can be demonstrated using a tunable $\mathrm{RF}$ MEMS resonator. The tunable resonator consists of an open-ended $\lambda / 2$ transmission-line resonator symmetrically loaded with two analog RF-MEMS varactors as shown in Fig. 7. The resonant frequency of this resonator is given by [7]

$$
\bar{B}_{p}-\cot \theta_{2}+\tan \theta_{1}=0
$$

where $\theta_{1}$ and $\theta_{2}$ are the equivalent electrical length of $l_{1}$ and $l_{2}$, and $\bar{B}_{p}$ is the normalized total shunt susceptance, which is mainly contributed by the varactors. An assumption that the tuning ratio remains 1.5 is made in the calculation of tuning range.

A 906- $\mu \mathrm{m}$ long coplanar-waveguide (CPW) of 50$\Omega$ is loaded with two analog RF-MEMS varactor at $l_{1}=100 \mu \mathrm{m}$. The model extracted from the previous measurements is applied to this resonator. In Fig. 8, region I shows the actual tuning range, while region II is based on the concept of the conventional spring constant. Because the varactor can not revert to the initial position, the actual tuning range is reduced by $60 \%$ compared to the conventional tuning range at the 1,370 hour.

\section{CONCLUSION}

An experiementally-extracted model for viscoelastic behavior in analog RF-MEMS devices is presented based on high-accuracy, high long-term stability measurement up to 1,370 hours. A new parameter named dynamic spring constant that can reveal the true electromechanical behavior of analog RF-MEMS devices is proposed. The impact of this behavior is demonstrated using a simulation of a tunable RF-MEMS resonator. This model and dynamic spring constant may improve the controllability of MEMS devices in long-term operation and lead to more reliable designs.

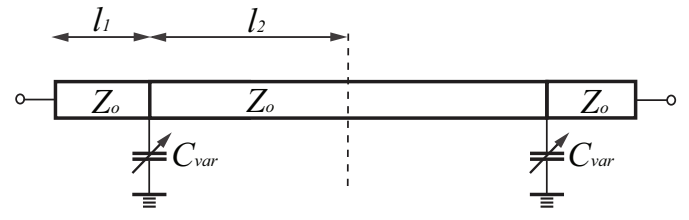

Figure 7. Schematic of the tunable resonator loaded with two RF-MEMS varactors.

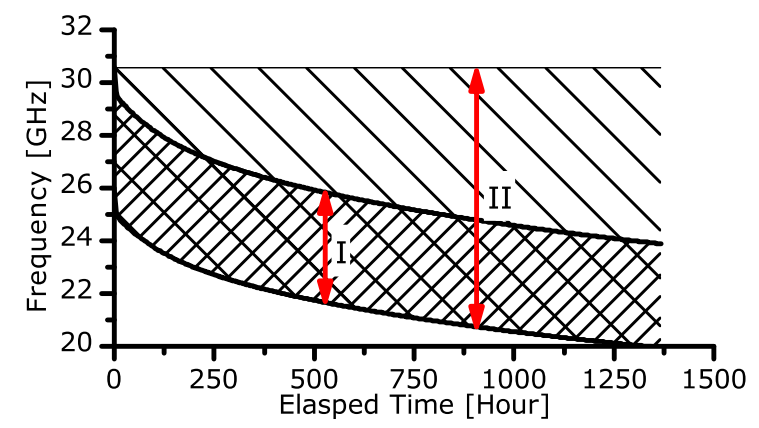

Figure 8. The simulated tuning range of the tunable RF-MEMS resonator. (I) The actual tuning range. (II) Based on conventional spring constant.

\section{ACKOWNLEDGE}

This material is based upon work supported by NNSA Center of Prediction of Reliability, Integrity, and Survivability of Microsystems, and Department of Energy under Award Number DE-FC5208NA28617.

\section{REFERENCES}

[1] X. Yan et al., "Anelastic stress relaxation in gold films and its impact on restoring forces in mems devices," J. Microelectromech. Syst., vol. 18, no. 3, pp. 570-576, Jun. 2009.

[2] I. Chasiotis et al., "Strain rate effects on the mechanical behavior of nanocrystalline au films," Thin Solid Films, vol. 515, no. 6, pp. 3183-3189, Feb. 2007.

[3] D. J. Vickers-Kirby et al., "Anelastic creep phenomena in thin metal plated cantilevers for mems," in Mat. Res. Soc. Symp., vol. 657, 2001, pp. EE2.5.1-EE2.5.6.

[4] M. van Gils et al., "Evaluation of creep in $\mathrm{rf}$ mems devices," in EuroSime, Apr. 2007, pp. 16.

[5] Ansoft maxwell 3d. Ansoft LCC. Version 12, 2008. [Online]. Available: http://www.ansoft. com

[6] W. Yin et al., "Creep behavior of nanocrystalline nickel at 290 and 373 k," Mat. Sci. Eng., vol. 301, pp. 18-22, 2001.

[7] A. Abbaspour-Tamijani et al., "A millimeterwave tunable filter using mems varactor," in IEEE MTT-S Int. Micro. Symp. Dig., Jun. 2003, pp. 1785-1788. 